\title{
AS PSICOLOGIAS: UMA ABORDAGEM DENOMINADA TERAPIA COGNITIVO-COMPORTAMENTAL
}

\section{ARTIGO ORIGINAL}

BROGNOLI, Maicol de Oliveira ${ }^{1}$

SANTOS, Sônia Alves dos ${ }^{2}$

BROGNOLI, Maicol de Oliveira. SANTOS, Sônia Alves dos. As psicologias: Uma abordagem denominada terapia cognitivo-comportamental. Revista Científica Multidisciplinar Núcleo do Conhecimento. Ano 05, Ed. 11, Vol. 22, pp. 152-168.

${ }^{1}$ Graduação em Psicologia pela Universidade do Extremo Sul Catarinense - UNESC em 2017, Especialização em Docência do Ensino Superior e EJA pela Faculdade da Região Serrana - FARESE em 2019, Especialização em Iridologia pela Faculdade Einstein em 2019, Especialização em Psicologia Clínica pela Faculdade da Região Serrana - FARESE em 2019, Especialização em AEE e Educação Inclusiva pela Faculdade da Região Serrana - FARESE em 2020, cursando Especialização em Psicologia Comportamental e Cognitiva pela Faculdade de Venda Nova do Imigrante - FAVENI, cursando Especialização em Psicologia Social pela Faculdade de Venda Nova do Imigrante - FAVENI, cursando Especialização em Psicologia Jurídica e Avaliação Psicológica pela Faculdade de Venda Nova do Imigrante - FAVENI e cursando Mestrado em Ciências Ambientais pela Universidade do Extremo Sul Catarinense - UNESC.

${ }^{2}$ Graduação em Psicologia pela Universidade do Extremo Sul Catarinense (2017), Especialização em Iridologia pela Faculdade Einstein (2019), Especialização em Neuropsicopedagogia pela Uniasselvi (2020) e Especialização em Docência do Ensino Superior pela Uniasselvi (2020). Pós-Graduanda em AEE e Educação Inclusiva pela FARESE, Pós-Graduanda em Psicologia Jurídica e Avaliação Psicológica pela FAVENI e Pós-Graduanda em Psicologia Cognitiva e Comportamental. 
Novembro de

2020.

ISSN:

2448-0959,

Link

de

acesso: https://www.nucleodoconhecimento.com.br/psicologia/as-psicologias

\section{RESUMO}

O termo terapia cognitivo-comportamental (TCC) pode ser utilizado para abarcar inúmeras técnicas que permeiam os nortes científicos da abordagem cognitiva e uma combinação de procedimentos comportamentais. Nessa junção, enquanto a psicologia comportamental é focada no entendimento do comportamento, que também está em função de circunstâncias ambientais, a psicologia cognitiva busca compreender os meios pelos quais os seres humanos formulam sua aprendizagem, sua percepção, como recordam e pensam. As intervenções da terapia comportamental são focadas principalmente na modificação das contingências, procurando assim, alterar o comportamento, já a terapia cognitiva (TC), tem como objetivo atuar sobre a estrutura e o funcionamento cognitivo dos pacientes. Com o aumento da utilização das TCCs nas últimas décadas, a literatura especializada no assunto vem apresentando um conjunto de modelos teóricos diferenciados, que podem ser considerados como "terapias em abordagem cognitivo-comportamental". Por isso, o objetivo deste trabalho foi apresentar uma breve discussão sobre o surgimento das TCCs com ênfase nos principais modelos teóricos e nas principais perspectivas atuais sobre o assunto. Com este levantamento bibliográfico pode-se verificar que a articulação de diferentes teorias favoreceu a construção de uma terapia cognitivo-comportamental adequada à sua época, com inúmeras técnicas que têm evidenciado eficácia nos processos terapêuticos. Isso pode proporcionar aos profissionais da área inúmeros veículos para lidarem com a variedade de demandas existentes, levando sempre em consideração o perfil cognitivo do transtorno, a fase da terapia e a conceitualização cognitiva específica do paciente tratado.

Palavras-Chave: Modelos teóricos, processo terapêutico, terapia cognitivocomportamental. 


\section{INTRODUÇÃO}

Frequentemente, os termos terapia cognitiva (TC) e terapia cognitivo-comportamental (TCC), são utilizados como sinônimos para representar psicoterapias com formulações no campo do modelo cognitivo. Entretanto, o termo terapia cognitivocomportamental também pode ser utilizado para descrever várias técnicas que combinam uma abordagem cognitiva e um conjunto de procedimentos comportamentais. Por isso, pode-se dizer que o termo terapia cognitivocomportamental é utilizada de forma mais ampla, e inclui tanto a terapia cognitiva padrão quanto combinações de estratégias cognitivas e comportamentais (BECK, 2005).

Enquanto a psicologia comportamental é focada no entendimento do comportamento, que, também está em função de circunstâncias ambientais, a psicologia cognitiva procura promover estudos que busquem a análise dos meios pelos quais os seres humanos desenvolvem seus processos de aprendizagem, de percepção, como recordam e pensam (BAUM, 2006; STERNBERG, 1996/2010).

Originada na década de 40, a terapia comportamental (também conhecida como terapia analítico comportamental), foi inspirada, principalmente, pela pesquisa de Burrhus Frederic Skinner em análises experimentais do comportamento. Os livros O Comportamento dos Organismos (1938) e Ciência e Comportamento Humano (1953) descrevem os pontos essenciais e são tidos como um manual básico da psicologia comportamentalista. Desta forma, o profissional que se utiliza da terapia comportamental leva em consideração algumas variáveis, como: os sentimentos, as atitudes, as emoções, as somatizações, os valores e pensamentos. Essas variáveis são vistas como dependentes e resultantes de diferentes circunstâncias, também denominada de contingências. As intervenções da terapia comportamental são focadas principalmente na modificação das contingências, procurando assim, alterar o comportamento (MARCAL, 2005). O estudo desenvolvido por Skinner, que também é conhecido como behaviorismo radical, resumidamente, preconiza que se tratando de comportamento, este último pode ser determinado e definido pela interação do organismo com o ambiente. 
Outra abordagem que influenciou no desenvolvimento das TCC's foi o estudo feito pelo pesquisador Aaron Beck. A terapia cognitiva, como é mais conhecida, se iniciou na década de 1950, quando o pesquisador também atuava como psicanalista. Com o objetivo de validar os princípios psicanalíticos, esse pesquisador, deu início ao uso do método científico (adotado pelo meio acadêmico) para a condução de suas pesquisas. Estudando o modelo psicanalítico da depressão, ele propôs um modelo alternativo, chamando-o de modelo cognitivo de depressão. A partir deste modelo, ele pôde evoluir os aspectos teóricos e práticos e propôs um novo sistema de psicoterapia, denominado terapia cognitiva (TC), que tinha como objetivo atuar sobre a estrutura e o funcionamento cognitivo dos pacientes (BECK, 1967; BECK et al., 1979)

Atualmente, o desenvolvimento de diversas estratégias terapêuticas devido ao aumento do conhecimento científico, vem auxiliando os profissionais da área a tratar vários quadros psicopatológicos como: transtornos psicóticos, transtornos de ansiedade, transtornos alimentares, transtornos relacionados à dependência química, transtornos de humor, entre outros. Além disso, o desenvolvimento de programas de tratamento interdisciplinares que propõe uma articulação entre profissionais da área de saúde, só trouxe benefícios no tratamento destes quadros. Isso é evidenciado quando se observa o aumento de publicações científicas na área da saúde sobre a eficácia das terapias cognitivo-comportamentais (TCCs) no tratamento de variados transtornos psicológicos (CABALLO, 2003; KNAPP, 2004).

Devido principalmente ao aumento da utilização das terapias cognitivocomportamental nas últimas décadas, a literatura especializada no assunto apresenta um conjunto de modelos teóricos diferenciados, que podem ser consideradas como "terapias em abordagem cognitivo-comportamental". Por isso, o objetivo do presente trabalho foi apresentar uma breve discussão elucidando a eclosão das terapias cognitivo comportamentais (TCCs), dando ênfase aos principais modelos teóricos e abordando as principais perspectivas recentes em torno do assunto.

Utilizou-se do método de pesquisa qualitativo, no qual, busca-se compreender a importância das TCCs e suas perspectivas recentes, que, conforme sua natureza vem a básica, com o intuito de gerar novos conhecimentos sobre está modalidade 
terapêutica, sendo uma pesquisa descritiva proporcionando maior familiaridade com o tema em questão. A presente pesquisa utilizou do procedimento bibliográfico, na qual, a amostragem foi composta por artigos científicos, livros e revistas científicas.

\section{DESENVOLVIMENTO}

Dando início a presente discussão em torno do assunto abordado por este trabalho, vale-se da necessidade de referenciá-lo com as conceituações do renomado estudioso Bernard Rangé (2001, p.35), ressalvando que:

A psicoterapia cognitivo-comportamental é uma prática de ajuda psicológica que se baseia em uma ciência e uma filosofia do comportamento caracterizada por uma concepção naturalista $e$ determinista do comportamento humano, pela adesão a um empirismo e a uma metodologia experimental como suporte do conhecimento e por uma atitude pragmática quanto aos problemas psicológicos.

\section{ORIGEM DA TERAPIA COGNITIVO - COMPORTAMENTAL}

O desenvolvimento das terapias cognitivo-comportamental teve início na década de 60, com a chamada "revolução cognitiva". Estudos nesta área foram iniciados por pesquisadores como Aaron Beck, Michael Mahoney e Albert Ellis (dentre outros), e constituíram um grande marco na terapia comportamental (CABALLO, 1996). Desde então, a terapia cognitivo-comportamental evoluiu muito e, contemporaneamente destaca-se dentre as abordagens centrais nas múltiplas áreas de atuação da psicologia, angariando aceitação e prestígio pelos resultados alcançados com suas práticas, e pelo modo singular de seu enfoque e direcionamento.

Desde seu surgimento, várias abordagens da TCC foram sendo desenvolvidas, com vários graus de aplicação e sucesso. Segundo Dobson e Dozois (2001), essas abordagens podem ser classificadas em três divisões principais: a) as terapias de habilidades de enfrentamento, que procuram estimular o desenvolvimento de habilidades que forneçam ao paciente subsídios para lidar com situações problemáticas; b) a terapia de solução de problemas, que enfatiza o desenvolvimento de estratégias que possibilitam o paciente lidar com diversas dificuldades pessoais; e 
c) as terapias de reestruturação, objetivando mostrar ao paciente que problemas emocionais são uma consequência de pensamentos mal adaptativos, e que estes pensamentos distorcidos podem ser reformulados, promovendo pensamentos adaptativos.

Há diversos modelos conceituais de modificação cognitivo-comportamental, dentre eles alguns que são importantes e que iremos abordar brevemente neste trabalho são: o treinamento de autoinstrução, a terapia de solução de problemas, o treinamento de inoculação de estresse, a terapia construtivista, a terapia do esquema e terapia comportamental dialética.

$\mathrm{Na}$ década de 70, Donald Meichenbaum deu luz a técnica treinamento de autoinstrução (MEICHENBAUM, 1993), que prioriza a relação entre autoinstrução verbal e o comportamento, estimulando tarefas graduais, modelagem cognitiva, a orientação do treinamento mediacional e auto reforço. Já o treinamento de inoculação de estresse, que também foi desenvolvido por Meichenbaum (MEICHENBAUM, 1985), é uma abordagem multicomponente de habilidades de enfrentamento que se baseia na premissa de que, quando a pessoa aprender a lidar com níveis leves de estresse ela se torna "inoculada" ou "imune" a situações com níveis mais altos de estresse.

A terapia de solução de problemas, também proposta no início da década de 1970 pelos pesquisadores D'Zurilla e Goldfried (D'ZURILLA; GOLDFRIED, 1971), tem como objetivo o treinamento de autocontrole. Por isso, essa terapia trabalha com o desenvolvimento de habilidades básicas de solução de problemas, aplicando-as em situações problemáticas reais (como por exemplo, habilidades preventivas e para o enfrentamento do estresse, da raiva, para a depressão e situações psicológicas que envolvem o câncer). Desta forma, esta terapia busca promover uma mudança generalizada do comportamento do paciente em tratamento (NEZU, 1986).

Outro tipo de terapia bastante importante é a terapia construtivista. Ela foi desenvolvida no início da década de 80 (GUIDANO; LIOTTI, 1983), e ao invés de lidar com o conteúdo do pensamento, tem por objetivo enfatizar o processo de pensamento 
e a geração de significado. Segundo Neimeyer (NEIMEYER, 1995) sua abordagem é "radicalmente divergente de uma perspectiva tradicional de terapia cognitiva".

Em relação a abordagens que foram desenvolvidas nos últimos anos, podemos citar a terapia do esquema, proposta por Jeffrey Young (YOUNG; KLOSKO; WEISHAAR, 2003), e a terapia comportamental dialética (DBT), desenvolvida por Marsha Linehan (LINEHAN, 1993). Ambas abordagens cognitivo-comportamentais emergiram e ampliaram-se tendo por base a originalidade do modelo conceitual inicial. Destacamse como exemplos de abordagens de TCC usadas para tratar indivíduos com psicopatologia mais grave, como o transtorno de personalidade borderline.

Como exemplificado anteriormente, o que a literatura entende como "terapias em abordagem cognitivo-comportamental" (TCCs) é representada por um amplo espectro de terapias diferentes (DOBSON; SCHERRER, 2004; BORBA, 2005). Neste sentido, a Terapia Cognitiva (TC), desenvolvida por Aaron Beck e a Terapia Racional Emotivo Comportamental (TREC), criada por Albert Ellis, representam as duas modalidades preponderantes das últimas décadas (DOBSON; SCHERRER, 2004).

A abordagem proposta por Aaron Beck (BECK, 1963; 1964), chamada de Terapia Cognitiva (TC), foi desenvolvida para explicar os processos psicológicos em uma situação de depressão, com o objetivo de provar a teoria freudiana de depressão como hostilidade retrofletida reprimida. Entretanto, ao invés de hostilidade e raiva, a pesquisa sobre os sonhos dos pacientes deprimidos mostrou um "senso de derrota, fracasso e perda" (BECK, 1961). Isso mostrou que os sonhos poderiam ser simplesmente a força refletida dos pensamentos dos sujeitos. Por isso,

Beck propôs que os sintomas de depressão poderiam ser explicados em termos cognitivos como interpretações tendenciosas das situações, atribuídas à ativação de representações negativas de si mesmo, do mundo pessoal e do futuro (a tríade cognitiva) (BECK, 1976 apud KNAPP; BECK, 2008, p.56).

Com isso, teve início uma abordagem que focava o tratamento em problemas presentes, ao invés de traumas escondidos do passado, e na análise de experiências psicológicas acessíveis, ao invés de inconscientes. (BECK, 1976; BECK et al., 1979). 
Knapp e Beck (2008) explicam que a estruturação hierárquica da cognição desde seu processo primário proposta por Freud (fora da consciência e baseada em fantasias e desejos) quanto em seu processo secundário (com acesso à consciência e embasada nos princípios de realidade objetiva) serviram de pilar para os fundamentos da TC, bem como o conceito de que os sintomas são baseados em ideias patogênicas (BECK, 1963). Já em seus treinamentos iniciais em psicanálise e mesmo no decorrer de sua carreira, Beck demonstrou profundo interesse para com a vertente dos neoanalistas, como Alfred Adler, Karen Horney, Otto Rank e Harry Sullivan. Esta vertente enfatizava a importância de entender e lidar com as experiências conscientes dos pacientes:

[...] bem como a necessidade de tratar os significados que os pacientes atribuem a eventos que acontecem em suas vidas. A teoria cognitiva, com seu foco nos processos intrapsíquicos, e não no comportamento observável, é mais um legado da teoria psicanalítica, embora os procedimentos terapêuticos sejam mais semelhantes à terapia comportamental (BECK, 1984 apud KNAPP; BECK, 2008, p.57).

Fundamentalmente a TC defende que a maneira como as pessoas percebem e processam a realidade terá influência sobre a forma como elas se sentem e se comportam. Assim, seu objetivo terapêutico visa a reestruturação e correção dos pensamentos distorcidos e o desenvolvimento de soluções para produzir mudança e melhoras nos transtornos emocionais. Por isso, a TC preconiza que existem pensamentos nas fronteiras da consciência que ocorrem de forma espontânea e rápida, e podem ser analisados como uma interpretação imediata de qualquer situação (BECK, 1976).

Os pensamentos automáticos se diferenciam do fluxo normal de pensamentos que podem ser observados no raciocínio reflexivo ou na livre associação, normalmente são aceitos como plausíveis, e tendo sua acurácia aceita como verdadeira. Grande parte das pessoas não detecta conscientemente a presença dos pensamentos automáticos, entretanto com o devido treinamento é possível começar a monitorá-los e identificá-los (KNAPP; BECK, 2008). Segundo Beck e Newman (2005) pode-se perceber um pensamento, aprimorar o foco e a avaliação sobre ele, bem como existe a possibilidade da identificação e reflexão sobre uma sensação como a dor. 
De acordo com Knapp e Beck (2008), as distorções cognitivas mais evidenciadas nos diferentes transtornos emocionais são: a catastrofização, quando a pessoa pensa que o pior de uma situação irá acontecer sem levar em consideração a possibilidade de outros desfechos; o raciocínio emocional (ou emocionalização), quando se presume que sentimentos são fatos e estes guiam a interpretação da realidade; polarização (pensamento tudo ou nada, dicotômico), quando a situação é vista em apenas duas categorias, mutualmente exclusivas; abstração seletiva (visão em túnel), quando se foca em apenas um único aspecto da situação, realçando a parte negativa; leitura mental, quando se presume (sem evidências) o que os outros estão pensando; rotulação, colocar um rótulo global e rígido em si, numa situação ou pessoa; minimização e maximização, quando situações positivas são minimizadas e as negativas são maximizadas; e imperativos, interpretar situações em termos de como deveriam ser ao invés de como realmente são (KNAPP; BECK, 2008).

Dryden; Ellis, (2001) comentam que a terapia racional emotiva comportamental (TREC), originada por um Albert Ellis, na opinião de muitos estudiosos representa uma das primeiras TCCs. Originada há mais de 45 anos, essa terapia aborda o chamado modelo $A B C$, sugerindo que cada experiência ou evento dispara $(A)$ crenças individuais (B), gerando consequências (C) emocionais, comportamentais e fisiológicas. Também se propõe 12 crenças irracionais básicas, que tornam-se em expectativas irrealistas ou absolutistas, sendo a base do transtorno emocional. Por isso, o objetivo terapêutico aqui é fazer um levantamento das crenças irracionais e, mediante questionamento, desafio e debate lógico-empíricos, pelo convencimento buscar modificá-las (DRYDEN; ELLIS, 2001).

\section{SEMELHANÇAS E DIFERENÇAS ENTRE OS TIPOS DE TCC'S}

As abordagens em TCC podem compartilhar bases comuns, contudo deve-se levar em consideração que existem diferenças substanciais em suas metodologias e procedimentos. As distintas bases teóricas que no início do desenvolvimento destas intervenções cognitivo-comportamentais estiveram presentes, explicam as referidas diferenciações. Ao comparamos Aaron Beck e Albert Ellis, provindos das bases psicanalíticas, com outros teóricos como Meichenbaum, Goldfried e Mahoney, que 
foram originalmente treinados em modificação do comportamento, percebemos a singularidade de seus constructos (KNAPP; BECK, 2008).

Segundo os pesquisadores Dobson e Dozois (2001), as abordagens atuais em TCC são semelhantes em três proposições fundamentais. A primeira é o papel de mediação da cognição, que propõe que a resposta do paciente pode ser afetada pela avaliação de eventos internos e externos e por um processamento cognitivo. A segunda proposição defende que a atividade cognitiva pode ser monitorada, avaliada e medida. E a terceira, elucida que a mudança comportamental pode ser mediada por essas avaliações cognitivas, evidenciando indiretamente esta mudança.

Dessa maneira, a TCC vai de encontro a tratamentos puramente comportamentais, onde a cognição não se destaca como variável explicativa importante no foco da intervenção. Isso fica claro quando as abordagens voltadas somente para a mudança de comportamento, como o modelo estímulo-resposta, são observadas. Assim, do mesmo modo, uma terapia voltada apenas para a mudança cognitiva também não é cognitivo-comportamental. Por isso, qualquer forma de terapia que não inclua modelo de mediação como componente importante do plano de tratamento, não pode ser classificada como uma TCC (DOBSON; DOZOIS, 2001).

Admitindo teoricamente a mediação cognitiva dos sintomas e comportamentos disfuncionais onde o progresso terapêutico se processa na modificação do pensamento e de crenças disfuncionais, pode-se acolher ai uma das principais características utilizadas para definir o que é uma TCC. Entretanto, os vários tipos de TCCs podem compartilhar outros diversos pontos em comum que são variáveis no campo teórico (DOBSON; DOZOIS, 2001). Uma TCC comumente comporta menor tempo em seus processos de tratamento, por exemplo depressões e transtornos de ansiedades leves podem perdurar 12-16 sessões, ainda 1-2 anos de tratamento para transtornos de personalidade e outros com cronicidade acentuada. Outro ponto é que geralmente as TCCs são aplicadas a problemas ou transtornos específicos, refletindo em esforço contínuo para documentar efeitos terapêuticos, estabelecer fronteiras terapêuticas e aprimorar a busca por um processo psicoterapêutico eficiente diante de um determinado problema. Nesta abordagem o paciente é o agente ativo de seu 
tratamento, onde na maioria das TCCs o modelo terapêutico pode ser ensinado e a lógica para a intervenção deve ser comunicada ao paciente. Encontra-se aqui um contraste em comparação a outras abordagens psicoterápicas (KNAPP; BECK, 2008).

Outro ponto em comum em relação a maioria das TCCs é em relação ao seu objetivo de desenvolver no paciente aprendizado diante do processo terapêutico no decorrer da terapia.

Além de superar os problemas na terapia e, assim, aprender a prevenir recorrências, os pacientes também aprendem habilidades terapêuticas que eles próprios podem aplicar com abrangência a uma gama de diferentes problemas em suas vidas Devido a esses fatores, pode-se dizer que na TCC, os pacientes tornam-se seus próprios terapeutas. (KNAPP; BECK, 2008, p. 56).

Dobson e Dozois (2001) explicitam que mesmo diante de inúmeras sobreposições de procedimentos, deve-se conceber que há de fato uma só abordagem cognitivocomportamental, como na mesma direção pode-se afirmar que existe uma só terapia psicanalítica. Fica compreendido que nas TCCs encontra-se uma vasta diferença em abordagens, entretanto ao direcionarmos nosso foco a TC desenvolvida por Beck, podemos percebe-la mais uniforme, agregando especificamente conjunto próprio de princípios, metodologias e técnicas.

Resumidamente, diante das TCCs coleta-se um conjunto de pressupostos em comum que as relaciona: "a atividade cognitiva influencia o comportamento; a atividade cognitiva pode ser monitorada e alterada; o comportamento desejado pode ser influenciado via mudança cognitiva" (DOBSON, 2006; DOBSON E SCHERRER, 2004 apud RONDINA, 2008, p. 5). Portanto, intitula-se como "TCCs, as terapias onde a mediação cognitiva ocorre e quando o plano de tratamento também abarca este tipo de mediação como componente relevante". (DOBSON; 2006; DOBSON E SCHERRER, 2004; KNAPP, 2004 apud RONDINA, 2008, p. 5). 


\section{CONCLUSÃO}

As teorias dizem muito de seus fundadores, de suas aspirações, de seu contexto histórico-cultural e de suas experiências. Com a terapia cognitivo-comportamental pode-se observar também sua variabilidade enquanto abordagem provinda de variadas fontes teóricas, estudiosos e metodologias que visam no decorrer do tempo, promover aproximações nos mais variados pontos de vista e estudos elaborados. Precisa ser encarada como uma abordagem em evolução, e como toda teoria e paradigma, está vinculada as vicissitudes de seu contexto temporal, histórico-cultural, demandas sociais, buscando assim, compreender o ser humano em sua multidimensionalidade biopsicossocial. Barbosa et al., (2014) auxiliam na conclusão de que a TCC surgiu de um panorama variável desde sua origem aos dias atuais. É fundamentada em teorias filosóficas milenares, bem como em teorias modernas e pós-modernas. Ai está a riqueza desta abordagem com suas "psicoterapias", pois todas estas fontes proporcionam teorias férteis em suas origens epistemológicas. Todo este contexto propicia o desenvolvimento de uma TCC articulada para sua época, apresentando técnicas testadas e aprovadas no sucesso dos processos terapêuticos. Os profissionais imersos nesta realidade, possuem ferramentas eficazes que Ihes habilitam a trabalhar com a variedade de demandas existentes.

Analisa-se que somente didaticamente é que diferencia-se intervenções de TC de um lado e técnicas da TCC do outro. Na prática, diversas técnicas podem influenciar os processos de pensamento. Do mesmo modo, muitas outras visam mudanças de comportamento. Assim, mediante ao cuidado e capacidade técnica para tal, o profissional habilitado pode combinar e cambiar técnicas diferenciadas. Devem estar inclusas na aprovação do rol científico da abordagem, considerando as nuances cognitivas do transtorno, o estágio da terapia, a conceitualização cognitiva e as singularidades do paciente.

\section{REFERÊNCIAS}

BARBOSA, Arianne de Sá; TERROSO, Lauren Bulcão; ARGIMON, Irani Iracema de Lima. Epistemologia da terapia cognitivo-comportamental: casamento, amizade ou 
separação entre as teorias?. Bol. - Acad. Paul. Psicol., São Paulo , v. 34, n. 86, p. 63-79, 2014 . Disponível em <http://pepsic.bvsalud.org/scielo.php?script=sci_arttext\&pid=S1415711X2014000100006\&lng=pt\&nrm=iso >. Acesso em: 23/11/2020.

BAUM, W. M. Compreender o behaviorismo. Porto Alegre: ArtMed, 2006. Disponível em: https://www.academia.edu/34222551/Compreender_o_Behaviorismo__William_Baum.pdf. Acesso em: 17/10/2019.

BECK, A. T.; WARD, C.H. Dreams of depressed patients: characteristic themes in manifest content. Arch Gen Psychiatry, n. 5, p. 462-467, 1961. Disponível em: 10.1001/archpsyc.1961.01710170040004. Acesso em: 20/10/2019.

BECK, A. T. Thinking and depression. I. Idiosyncratic content and cognitive distortions. Arch Gen Psychiatry., n. 9, p. 324-333, 1963. Disponível em: https://jamanetwork.com/journals/jamapsychiatry/article-abstract/488402. Acesso: 18/10/2019.

BECK, A. T. Thinking and depression: II theory and therapy. Arch Gen Psychiatry., n. 10, p. 561-571, 1964. Disponível em: 10.1001/archpsyc.1964.01720240015003. Acesso em: 18/10/2019.

BECK, A. T. Depression: Clinical, Experimental, and Theoretical Aspects. New York, NY: Harper and Row, 1967.

BECK, A. T. Cognitive Therapy and the Emotional Disorders. New York: International Universities Press, 1976.

BECK, A. T. Cognitive therapy, behavior therapy, psychoanalysis, and pharmacotherapy: a cognitive continuum. In: Williams JBW, Spitzer RL. Psychotherapy research: where are we and where should we go? New York: Guilford, 1984. 
BECK, A. T; NEWMAN, C. F. Cognitive therapy. In: Kaplan, Sadock's. Comprehensive textbook of psychiatry. 8th ed. New York: Lippincott Williams and Wilkins, 2005.

BECK, A. T.; RUSH, A. J.; SHAW, B. F.; EMERY, G. Cognitive therapy of depression. New York: Guilford; 1979.

BORBA, A. Disciplina Online de TCC: expansão das fronteiras da formação em TCC através da educação online. Rio de Janeiro, UFRJ. Dissertação de Mestrado, 2005. Disponível em: http://livros01.livrosgratis.com.br/cp023837.pdf. Acesso: 19/10/2019.

CABAllo, V. E. Manual de Técnicas de Terapia e Modificação do Comportamento. São Paulo: Livraria Santos Editora Com. Imp. Ltda., 1996. Disponível:

https://www.academia.edu/8978425/Caballo_V._E._1996_._Manual_de_Técnicas_d e_Terapia_e_Modificação_do_Comportamento. Acesso: 18/10/2019.

CABALLO, V. Manual para o tratamento cognitivo-comportamental dos transtornos psicológicos: transtornos de ansiedade, sexuais, afetivos e psicóticos. São Paulo: Santos, 2003.

DOBSON, K. S., et al. Manual de terapias cognitivo comportamentais. Porto Alegre: Artmed, 2006.

DOBSON, K. S.; DOZOIS, D. J. Historical and philosophical bases of the cognitivebehavioral therapies. In: Dobson KS, editor. Handbook of cognitive-behavioral therapies. 2nd ed. New York: Guilford Press, 2001.

DOBSON, K. S.; SHCERRER, M. C. História e futuro das terapias cognitivocomportamentais. IN: Knapp, P. et al. Terapia Cognitivo-Comportamental na Prática Psiquiátrica. Porto Alegre: Artmed, p. 42-57, 2004. 
DRYDEN, W.; ELLIS, A. Rational emotive behavior therapy. In: Dobson KS, editor. Handbook of cognitive-behavioral therapies. 2nd ed. New York: Guilford Press, 2001.

D'ZURILLA, T. J.; GOLDFRIED, M. R. Problem-solving and behavior modification. J Abnorm Psychol., n. 78 , p. 107-126, 1971. Disponível em: http://dx.doi.org/10.1037/h0031360. Acesso em: 18/10/2019.

GUIDANO, V.F.; LIOTTI, G. Cognitive processes and emotional disorders: a structural approach to psychotherapy. New York: Guilford; 1983. Disponível em: https://doi.org/10.1093/sw/29.1.81. Acesso: 18/10/2019.

KNAPP, P. Princípios fundamentais da terapia cognitiva. In: Knapp, P. et al. Terapia Cognitivo-Comportamental na Prática Psiquiátrica. Porto Alegre: Artmed, p. 42$57,2004$.

KNAPP, P.; BECK, A. T. Fundamentos, modelos conceituais, aplicações e pesquisa da terapia cognitiva. Rev. Bras. Psiquiatr., v. 30, p. 54-64, 2008. Disponível em: http://www.scielo.br/pdf/rbp/v30s2/a02v30s2.pdf. Acesso: 20/10/2019.

LINEHAN, M. Cognitive-behavioral treatment of borderline personality disorder. New York: Guilford Press; 1993.

MARCAL, J. V. S. Estabelecendo objetivos na prática clínica: quais caminhos seguir? Revista Brasileira de Terapia Comportamental e Cognitiva. v. 7, n. 2, p. 231-146, 2005. Disponível em: https://doi.org/10.31505/rbtcc.v7i2.32. Acesso em: $19 / 10 / 2019$

MEICHENBAUM, D. H. Stress inoculation training: a clinical guidebook. New York: Pergamon Press, 1985.

MEICHENBAUM, D. H. Stress inoculation training: a twenty-year update. In: Woolfolk, R. L.; Lehrer, P. M., editors. Principles and practice of stress management. New York: Guilford Press, 2007. Disponível em: https://i-cbt.org.ua/wp- 
content/uploads/2017/11/2007-Principles-and-practice-of-stress-managementLehrer-Woolfolk-Sime.pdf. Acesso em: 20/10/2019.

NEIMEYER, R. A. Constructivist psychotherapies: features, foundations and future directions. In: Neimeyer, R. A., Mahoney, M. J., editors. Constructivism in Psychotherapy. Washington, DC: American Psychological Association, 1995.

NEZU, A. M. Efficacy of a social problem solving therapy approach for unipolar depression. J Consulting Clin Psychol., v. 54, n. 2, p. 196- 202, 1986. Disponível em: https://psycnet.apa.org/fulltext/1986-20326-001.html. Acesso em: 20/10/2019.

RANGÉ, B. Psicoterapia Comportamental e Cognitiva: Pesquisa, Prática, Aplicações e Problemas. Volume 1. São Paulo: Editoria Livro Pleno, 2001.

RONDINA, R. C. Terapia Cognitivo Comportamental: desenvolvimento histórico, tendências atuais. Revista Científica Eletrônica de Psicologia, v. 1, p. 1 - 6, 2008.

STERnBERG, R. J. Psicologia Cognitiva (M. R. B. Osório, trad.) Porto Alegre: Artmed, 2008.2 Disponível em: https://www.academia.edu/16543597/Sternberg._R._J._2008_._Psicologia_Cognitiva . Acesso em: 19/10/2019.

YOUNG, J.; KLOSKO, J.; WEISHAAR, M. E. Schema therapy: a practitioner's guide. New York: Guilford, 2003. Disponível em: https://www.guilford.com/excerpts/young.pdf?t. Acesso em: 20/10/2019.

Enviado: Novembro, 2020.

Aprovado: Novembro, 2020. 